\title{
"Buenos Aires, qué ciudad más acogedora". Racialización y sexualización de sudamericanas en sites destinados a turistas sexuales
}

\author{
Adriana Piscitelli ${ }^{1}$
}

\begin{abstract}
SÍNTESIS
Tomando como referencia el material diseminado en web sites destinados a turistas sexuales heterosexuales, en este texto examino en una lectura antropológica la inclusión de "nuevos" lugares de América del Sur en los circuitos globales de esos viajantes. Mi argumento principal es que esa integración está vinculada a factores económicos, politicos y culturales que se expresan en procesos de racialización diferenciados mediante los que mujeres de diferentes países y regiones, lejos de ser homogeneizadas, son insertadas en una jerarquía erótica. Describo las características del espacio virtual contemplado, de sus usuarios, sus interacciones y las prácticas sexuales por las que se interesan, creando un contexto para analizar en una perspectiva comparativa las imágenes femeninas difundidas en el site. En la conclusión considero los aspectos utilizados para tornar algunos países y ciudades de América del Sur, particularmente Buenos Aires, un atractivo espacio de consumo sexual.
\end{abstract}

\begin{abstract}
Using as reference the material appearing in web sites aimed at heterosexual sex tourists, in this study I examine, from an anthropological viewpoint, the inclusion of "new" places in the sex tourism circuits in South America. My main argument is that this integration is linked to economic, political and cultural factors that are expressed in differentiated racialization processes by means of which women of different countries and regions, far from being homogenized, are placed in an erotic hierarchy. I describe the characteristics of this virtual universe, of its users, their interactions as well as the sexual practices they are interested in so as to create a context to make a comparative analysis of the feminine images disseminated in the site. As a conclusion, I take into consideration the aspects used to make some South American countries and cities, particularly Buenos Aires, an attractive venue for sex consumption.
\end{abstract}

Palabras clave: Turismo sexual - internet - género - raza - nacionalidad.

Key words: Sex tourism - internet - gender - race - nationality. 


\section{Introducción}

En este texto tomo como referencia el material diseminado en sites destinados a turistas sexuales heterosexuales para reflexionar sobre la inclusión de "nuevos lugares", en América del Sur, en los circuitos globales de esos viajeros. En la literatura sobre la transnacionalización del mercado sexual, la asociación entre mujeres de ciertas regiones pobres del mundo y el sexo comercial es vinculada a tres factores: los viajes de los turistas de los países ricos para consumir sexo en el "Tercer Mundo", ${ }^{2}$ los desplazamientos de personas del "Sur", principalmente mujeres, para trabajar en night clubs y burdeles en los países del "Norte" y la Internet. ${ }^{3}$ En esa producción, las páginas web son consideradas espacios primordiales para la producción y diseminación de estereotipos sexualizados y racializados de esas mujeres ${ }^{4}$ (Brennan, 2004; Kempadoo, 2004; Bishop y Robinson, 2002). Compartiendo esa percepción, algunos estudios sobre la articulación entre turismo e industria del sexo en el Caribe y en Tailandia prestan atención al material difundido en esos web sites (Brennan, 2004; Kempadoo, 2004; Bishop e Robinson, 2002; Rivers-Moore, 2009). ${ }^{5}$

Diferentemente de esos estudios, que utilizan material de esos sites para completar las declaraciones de turistas sexuales sobre algún país determinado, en este texto me centro exclusivamente en una página web que disemina material sobre diferentes partes del mundo: el World Sex Archives. ${ }^{6}$ Analizando las imágenes de mujeres producidas en ese site en una lectura antropológica expongo mi argumento principal: si bien la íntima asociación entre turismo sexual y aspectos económicos es innegable, el surgimiento de nuevos destinos está vinculado también a factores políticos y culturales que se expresan en procesos de exotización y racialización diferenciados mediante los que las mujeres de diferentes países y regiones, lejos de ser homogeneizadas, son insertadas en una jerarquía erótica. ${ }^{7}$

La literatura internacional sobre turismo sexual permite percibir que la apreciación de los destinos escogidos por los viajeros en busca de sexo se altera durante el tiempo. Personas locales que fueron blanco de ese tipo de turistas durante décadas, están perdiendo valor en el mercado del sexo. En esa producción se considera que en las décadas de 1950 y 1960 las mujeres del 
Sudeste de Asia y de Asia oriental (regiones intensamente buscadas para el consumo del sexo a partir de ese período), representaron el ideal de mercancía erótica, deseadas por la promiscuidad y pasividad que se les atribuía. En el final del siglo XX, esas regiones habrían perdido "valor". Según la autora australiana Berverly Mullings (1999), como otros tipos de turismo, el turismo sexual está marcado por la búsqueda de experiencias singulares que, concediendo un plus de valorización a los turistas, otorgue a esos viajantes una cierta distinción social, diferenciándolos de los turistas "masificados". En este sentido, algunos centros asiáticos, como Tailandia o Filipinas, excesivamente populares, habrían llegado a un "punto de saturación". La presencia de un número creciente de viajeros que acuden a esos países con el objetivo del consumo de sexo tornarían esos paisajes sexuales menos auténticos, menos reales y, por lo tanto, menos deseables.

A partir de la década de 1980, la circulación de turistas en busca de sexo se dirige a otros escenarios habitados por "nuevos" seres apetecibles para el consumo de sexo, todavía más exóticos, todavía más auténticos y, por lo tanto, más eróticos. En ese movimiento, nuevos lugares se tornan destinos deseados en América del Sur. La inserción del Nordeste del Brasil en esos circuitos en la década de 1990 y, algunos años más tarde, de Buenos Aires y otras ciudades de Argentina, ofrece ejemplos de las alteraciones de esos circuitos.

Retomando mi argumento, basándome en el web site analizado considero que la producción de nuevos "lugares" destinados al turismo sexual obedece a la lógica de la industria de turismo global. Algunos autores afirman que la particularidad de esas páginas web en relación a la industria de viajes reside en que ellas no venden apenas productos y servicios, lugares, gente y aventura sexual, sino que los venden a través de discursos de mercado, género, raza y sexualidad (Chow-White, 2006). Compartiendo esa idea, afirmo que, a pesar de existir una relación íntima entre turismo sexual y desigualdad, la pobreza, ni siquiera cuando es extremada, garantiza el éxito de un nuevo lugar. En el marco de ciertas condiciones económicas, aspectos culturales que se expresan en la intersección entre trazos raciales, étnico/ nacionales, regionales y estilos de sexualidad operan como atracción para el surgimiento de nuevos blancos para ese tipo de turismo. La atribución de esos trazos es construida en un marco en el que la recreación de códigos de la sexualidad es orientada por 
una educación colectiva, a través de la transmisión de normas de género y saberes que, atravesados por género, trazan fronteras etnosexuales. Se trata de límites entre grupos, caracterizados por la intersección e interacción entre sexualidad y etnicidad (Nagel, 2003). Esas fronteras, que son fundamentales en la delimitación de espacios turísticos para el consumo de sexo, muestran el surgimiento de nuevos procesos de racialización.

Delineo, en primer lugar, un contexto para analizar las imágenes diseminadas en el web site, describiendo las características del espacio virtual contemplado, de sus frecuentadores, sus interacciones y las prácticas sexuales por las que se interesan. Analizo después, en una perspectiva comparativa, las imágenes difundidas sobre las mujeres de América del Sur. En la conclusión considero las categorizaciones utilizadas en el web site para tornar exóticas a las mujeres de ciertos lugares, particularmente las de Argentina y Brasil. En la conclusión, retomo mi argumento inicial, reflexionando sobre como los procesos de racialización que operan en ese espacio participan en las alteraciones de la geografía mundial del turismo sexual.

\section{Contextos}

Las investigaciones en el ciberespacio han adquirido legitimidad en el ámbito de la antropología (Escobar, 1994; Strathern, 1994; Guimarães, 2000; Hine, 2000). Sin embargo, las discusiones de la disciplina no dejan de mostrar la inquietud suscitada, sobre todo, por la idea de una etnografía en espacios virtuales. Este debate trata de problemas éticos y metodológicos ${ }^{8}$ y presenta consideraciones sobre trabajo de campo y sobre la concepción de etnografía, particularmente en lo que se refiere al objetivo de develar un contexto a través de una descripción densa (Cavanagh, 1999; Wittel, 2000). Manifestando mi acuerdo con Escobar (1994) y con Guimarães Jr (2000) en lo que refiere a afirmar que el espacio virtual es una de las esferas constitutivas de las sociedades complejas, considero que si la idea de una etnografía de esos lugares virtuales está abierta a la discusión, es innegable que una lectura antropológica de esos espacios hace sentido. Esa lectura se torna especialmente válida cuando se considera que cabe a la disciplina delinear los mapas de significado 
vinculados a las diferentes configuraciones sociales (sin perder de vista los procesos de interacción que existen en ellas) y llevando en cuenta que el ciberespacio posibilita la formación de nuevas redes, con referencias específicas.

En este artículo pienso el ámbito del site a la manera de un micro-contexto, a la manera en que son accionadas ciertas concepciones sobre diversas regiones del mundo. Pero este espacio no puede ser separado del proceso amplio que posibilita su creación: el movimiento creciente de cruzar las fronteras para vender o para consumir servicios sexuales (Thorbek, 2002). Los media electrónicos son parte constitutiva de este proceso, así como de otros vinculados al "nuevo orden global" (Appadurai, 1996). En lo que se refiere al análisis del World Sex Archives, esta particularidad convierte la división virtual/real en algo artificial, un aspecto que es evidente cuando se consideran las dinámicas establecidas on y off line, dentro y a partir de este espacio virtual. Comprender el significado de las nociones accionadas en el site exige situar el material en relación a los diversos aspectos de ese proceso.

Los análisis sobre el turismo sexual muestran la heterogeneidad presente en el universo de consumidores en lo que se refiere al tipo de relaciones establecidas con las mujeres nativas de los países del "Sur". En esa literatura se crearon diversas categorías para tratar esas diferencias. Las denominaciones otorgadas a los diferentes "tipos" de viajeros en busca de sexo varían de un autor a otro, pero hay una relativa convergencia en señalar que para algunos el turismo sexual amplía el abanico de opciones disponibles en lo que se refiere a relaciones sexuales con cierta durabilidad y no exenta de sentimientos. Para otros, al contrario, este tipo de turismo permite innumerables y efímeras experiencias sexuales (Davidson, 1996; Silva e Blanchette, 2005; Seabruck, 2001; Piscitelli, 2004; 2006). Considerando las dimensiones trazadas por Luiz Fernando Dias Duarte (2004) en la configuración de la sexualidad moderna, esos viajeros parecen corporeizar una expresión aguda del hedonismo, buscando un placer enteramente desvinculado de inversiones afectivas.

Los viajeros que corresponden a esa última categoría son, precisamente, los principales usuarios del web site analizado. Destaco este punto, imposible de aprender mediante un estudio centrado exclusivamente en esas páginas web, subrayando la importancia de no generalizar esas observaciones a todos los 
turistas sexuales. Los usuarios del web site constituyen un tipo particular, extremo, hard core, entre esos viajeros. Sin embargo, ellos son importantes en la ampliación y modificación de los circuitos de turismo sexual internacional y, en este sentido, es importante considerar el singular efecto amplificador de la web.

La frecuencia impresionante de mensajes de los mismos usuarios sugiere una relación casi obsesiva con el tema de esos viajes. Ellos son ávidos consumidores de información que permita ampliar el abanico de experiencias sexuales teñidas por marcas étnicas. Sin duda, no constituyen una comunidad en el sentido concedido tradicionalmente a ese término, es decir, conformaciones de agentes que comparten un origen, una localidad con límites geográficos establecidos, vinculados por relaciones que involucran circuitos de reciprocidad. Sin embargo, a pesar de su heterogeneidad y de su localización dispersa, esos viajeros comparten características distintivas en función de las que establecen cierto tipo de intercambios.

La página inicial del World Sex Archives (WSA) muestra una amplia imagen con los rostros de mujeres de las más diversas características, anunciando la diversidad étnica y racial presente en el material que circula en él. El web site se presenta como un espacio diferenciado en relación a otros dedicados al sexo y a la pornografía, cuya singularidad reside en ofrecer informaciones útiles para viajeros. El punto de partida es una comparación, replicada recurrentemente en los mensajes, en la que las nativas del "Sur" aparecen dotadas de una inocencia natural, posible de ser corrompida por el ejemplo de las "Occidentales".

Las mujeres de los países occidentales son perras malcriadas. Reciben demasiado y dan muy poco. Saben que pueden tratar a sus hombres como si fueran basura. ¿Sabes por qué una mujer sonríe el día de su casamiento? Porque sabe que no tendrá que hacer sexo oral con él. ${ }^{9}$

World Sex Archives preview

Me alegro de que haya una frontera entre América del Norte y Sudamérica, porque no quisiera que las mujeres americanas corrompan a las latinas, que son un verdadero trabajo de arte y merecen ser amadas y bien tratadas. ${ }^{10}$

18/10/2003, Línea de conversación: "Attitudes, changes and the Latin AAA Farm System" / Argentina. 
El material exhibido en el preview del site alude a la diversidad de preferencias de los turistas sexuales. Sexo barato es uno de esos aspectos, pero hay otros, como la demanda de creación de nuevos espacios para los viajeros que buscan sexo. Se solicita a los usuarios informaciones sobre áreas todavía no "descubiertas". Esa lógica conduce al rechazo de los lugares con exceso de gringos (overgringofied) y muestran la valorización de los espacios frecuentados solo por habitantes locales, considerados más "auténticos". Los lugares poco explorados parecen fascinar por el carácter de aventura y desafío que presentan.

El WSA está constituido por diversos espacios: un área de mensajes, en la que son listados en orden cronológico, un área de fotografías, otra que aglutina los archivos completos organizados por país, un área de chat y un área destinada al calendario de eventos mensuales, en el que son anunciadas fiestas con acompañantes en diversos lugares del mundo y las fechas en las que los usuarios estarán viajando por lugares determinados, ofreciéndoles la oportunidad de encontrarse. Concentré la colecta de material en el área de archivos por país, que incluyó los intercambios de mensajes disponibles en el site entre noviembre de 2003 y agosto de 2005 que remitían a ese período y a años anteriores.

Recogí material relativo a diversos países siguiendo ciertos criterios. Orientada por el interés en comprender como algunas regiones pobres del mundo atraen turistas sexuales, en cuanto otras también pobres y relativamente próximas no lo hacen, e interesada en entender la dinámica de la circulación de esos viajeros, recogí de manera extensiva material (texto y fotografías) relativo a varios países de América del Sur. Las fotografías son aspectos importantes en los intercambios de mensajes. Los usuarios que las obtienen parecen obtener un plus de valorización en el web site. Muchos tratan de negociar al mismo tiempo el precio del encuentro sexual y el derecho a fotografiar a las mujeres.

Las imágenes sugieren especificidades en lo que se refiere a estilos de corporalidad y sexualidad. Las fotografías de africanas las retratan, frecuentemente, vestidas, a veces con trajes étnicos y exhibiendo posturas no erotizadas. Las mujeres asiáticas aparecen vestidas con ropas occidentales, semidesnudas o sin ropas, sonrientes, exhibiendo ocasionalmente gestos marcados por la etnicidad, tales como las manos, palma contra palma, pegadas al pecho. Al contrario, las mujeres del Caribe y de América del Sur 
son presentadas en posturas corporales extremadamente sexualizadas. A ellas les toca exhibir los genitales y/o ser mostradas en situaciones explícitas de relacionas sexuales o masturbación. Ese tipo de imágenes está vinculado sobre todo a los países más "tropicalizados" (Aparicio y Chavez-Silverman, 1997) como el Brasil, o en los que el sexo es percibido como más banal y barato, como República Dominicana y el Paraguay, en el que hay una verdadera colección de fotografías ginecológicas, con la explicación adicional de que las mujeres de ese país adoran posar de esa manera para las cámaras.

En el período de recolección del material del web site, América del Sur concentraba $20 \%$ de los mensajes sobre las regiones pobres del mundo. ${ }^{11}$ Apenas $1 \%$ de esos mensajes se refería a África, 28\% a América Central y el Caribe y 51\% a Asia. Sin embargo, es importante considerar que Asia es un continente en el que el turismo sexual tiene una historia de varias décadas. En países como Tailandia, Corea del Sur y Filipinas, el sexo comercial direccionado al turismo se desarrolló masivamente teniendo como antecedente la atención de soldados de los Estados Unidos durante las guerras de Corea, Indochina y Vietnam ${ }^{12}$ (Michel, 1998; Enloe, 1989). En América del Sur la problemática es detectada, sobre todo, a partir de la década de 1990.

En el ámbito de esa región elegí países que presentan ciertas especificidades. Algunos, por ser considerados ya espacios tradicionales de atracción, como Brasil y Colombia. Otros, como Argentina, por ser considerados los espacios más novedosos de atracción para los turistas que viajan buscando sexo. Finalmente elegí otros espacios, por ser considerados destinos menos atractivos, como Bolivia. La popularidad de ciertos países se torna evidente considerando el volumen de material sobre ellos: en el período analizado, el intercambio de mensajes sobre Chile, Uruguay, Bolivia, Perú y Paraguay no superó los 122 mensajes. El material sobre Argentina fue 10 veces mayor. El material sobre Colombia cuadriplicó el volumen de intercambios de mensajes sobre Argentina y el material sobre Brasil prácticamente cuadruplicó el número de mensajes sobre Colombia. El volumen diferenciado de material fue utilizado a la manera de puerta de entrada para explorar los aspectos vinculados a la geografía mutante del turismo sexual. El análisis fue realizado contrastando el material de esos países entre si y creando una perspectiva 
comparativa más amplia a través de la lectura de material relativo a los dos países más "populares" del Caribe, República Dominicana y Cuba y a países que estaban entre los más y menos buscados en Asia y Âfrica: Tailandia, Mongolia y Sri-Lanka; Kenia y Tanzania.

\begin{tabular}{|c|c|c|}
\hline País & $\begin{array}{l}\text { No de líneas de } \\
\text { conversación/tópicos }\end{array}$ & $\begin{array}{l}\mathbf{N}^{\circ} \text { de mensajes } \\
\text { integrados en los tópicos }\end{array}$ \\
\hline Brasil & 1912 & 22339 \\
\hline Argentina & 164 & 1223 \\
\hline Bolivia & 5 & 22 \\
\hline Chile & 12 & 62 \\
\hline Colombia & 439 & 4780 \\
\hline Ecuador & 22 & 122 \\
\hline Guyana & 2 & 16 \\
\hline Paraguay & 7 & 121 \\
\hline Perú & 41 & 362 \\
\hline Uruguay & 5 & 25 \\
\hline Venezuela & 38 & 362 \\
\hline Total & 2647 & 29434 \\
\hline
\end{tabular}

América del Sur: Líneas de conversación y mensajes. Fuente: www.worldsexarchives.com, julio de 2005.

\section{Viajeros}

Los intercambios de mensajes posibilitan trazar algunas de las características que los usuarios se atribuyen: edad, nacionalidad, profesión, estado civil. Se presentan con edades diversificadas (20, 30, 40, 50 años); generalmente originarios de países "ricos", a pesar de que entre ellos hay nativos de los países del "Sur", peruanos, brasileños, argentinos, cubanos, que residen hace tiempo en el Norte y de países que, a pesar de europeos, no son considerados ricos, como Rumania. Mostrando la hibridez que permea la categoría "turista", inclusive cuando se trata de aquellos que viajan en busca de sexo (Agustin, 2007), 
los usuarios se consideran viajeros de vacaciones o de negocios que estiran por algunos días el viaje, o expatriates, ciudadanos de países "ricos" residentes en el "Sur", entre ellos jubilados cuyas pensiones les posibilitan llevar una vida confortable y plena, que además les permite pagar encuentros sexuales en países del Tercer Mundo. Se presentan como solteros recalcitrantes, novios, viudos recientes o casados. Esos últimos expresan la conciencia de mantener una doble vida, actualizando en alguna medida y en escala internacional, las relaciones que, en el pasado, los hombres tenían con amantes en sus lugares de origen.

El conjunto de mensajes sugiere distinciones que atraviesan el universo de usuarios, pero la "identidad" creada a través del objetivo común compartido por esos viajeros parece diluir las diferencias entre ellos. Compartiendo un interés común (el consumo de sexo ofrecido por mujeres del "Sur"), los usuarios del site utilizan un lenguaje diferenciado. El idioma es el inglés. En el marco de un dominio desigual de ese idioma, los viajeros se comunican utilizando una terminología específica para referirse a sí mismos, a los estilos de mujeres con los que entran en contacto y a las prácticas sexuales. El término mongers, con el que se autodenominan, es una expresión de ese lenguaje. En inglés, esa palabra solo adquiere sentido acompañada por otra, un fishmonger es un vendedor de pescado. En el web site la palabra monger no acompaña otra. La parte implícita puede ser sexo o prostitutas (sex/whore) mongers. Los usuarios se refieren a una diversidad de tópicos: las mujeres, aludiendo al grado de profesionalidad y a la disponibilidad para pasar bastante tiempo con los viajeros entablando relaciones a la manera de novios (girl friend experience) e, inclusive, viajar con ellos, en el país de origen o para el exterior; las prácticas sexuales, $B B, B J$ y $B B B J$ son siglas que aluden al $b a-$ reback, práctica de mantener relaciones sexuales sin preservativo, al blow job, al sexo oral y al bare back blow job, al sexo oral sin preservativo.

Esos hombres crean corredores a través del mundo en los que circulan y se encuentran, establecen intercambios y se hacen favores mutuos. Para ellos, esa página web, conectando personas que antes de participar en él se sentían aislados o inclusive, perversos, opera a la manera de espacio de legitimación colectiva del interés por el consumo de sexo a través de las fronteras. De acuerdo con uno de ellos: 
Hace dos años yo consideraba esto como un mal necesario. Era una actividad que tenía que disfrutar solo, no podía hablar sobre ella con otras personas. Otros hombres conversan sobre fútbol o automóviles, yo pensaba en conchas. Comencé a participar en este grupo de discusión cuando fui por primera vez a Río. Cambié de manera dramática, sabiendo que hay un lugar en el mundo con probablemente decenas de miles de bellas chicas que trabajan y miles de compañeros como yo. ${ }^{13}$

14/12/2002, Línea de conversación: "Is there is life after Rio" / Brazil

La heterosexualidad de la página web es marcada en los mensajes, en los que hay evidente rechazo a los travestis, denominados transtesticles, o impostores, como si relacionarse con ellos contaminase el estilo de masculinidad difundido en el site que, al contrario aparece reforzada por el consumo de sexo ofrecido por mujeres de "otros" lugares. Cuando los mongers se encuentran con mujeres del "Primer Mundo" involucradas en ese tipo de actividad, parecen sentirse desconcertados. Es el caso de un turista sexual circulando por Kenia.

Vi algo interesante en la playa Diani, en la costa, al sur de Mombasa. Vi dos europeas (una era extremadamente atractiva) con hombres prostitutos, hombres Masai, negros, altos, vestidos con ropa tradicional. Si hubiera estado con mi máquina les hubiera sacado fotos y las hubiera subido al web site. Ya había leído sobre eso, pero fue la primera vez que lo vi en la vida real. ${ }^{14}$

12/01/2001, Línea de conversación: “Kenya” / Nairobi

La construcción de ese estilo de masculinidad exige la inferiorización de las mujeres, y también la constitución de una superioridad considerada "Occidental" en relación a las masculinidades "nativas", que se expresa a través de la comparación entre diferentes aspectos. En lo que se refiere a los nativos de África, el punto de comparación son las prácticas sexuales, consideradas más sofisticadas en los Occidentales y más "básicas" entre los africanos. En términos de los asiáticos, esa superioridad se expresa en el tamaño del pene y en el grado de potencia y exigencia sexuales. En el site, el color blanco es utilizado, muchas veces, como sinónimo de Occidental. Es un aspecto marcado, sobre todo, cuando se trata de los pocos usuarios que se 
presentan como negros. El color blanco es innegablemente considerado como atributo vinculado a la superioridad de esos viajeros sobre las poblaciones nativas y/o sobre viajeros y clientes no Occidentales. El site opera como un espacio de producción y transmisión de "saberes" en lo que se refiere a masculinidades e femineidades, delineadas a través de prácticas sexuales, estilos de comportamiento y de atributos vinculados a las mujeres.

\section{Intercambios}

El intercambio de mensajes abarca asuntos de interés de todos los turistas: precio y calidad de los hoteles, obtención de pasajes baratos, calidad de los restaurantes, con especial atención en la "autenticidad" de la comida. Los mensajes revelan el interés por la situación política y económica de los diversos países y, también, aspectos vinculados a las leyes sobre prostitución, sobre todo, con menores de edad que, ocasionalmente, revelan criterios morales.

De cualquier modo muchachos, aunque la edad de consentimiento legal fuera 5 años, creo que es inmoral pagar para hacer sexo con chicas menores de 18 años... por la simple razón de que una chica con menos años no tiene condiciones de decidir libremente si quiere hacer sexo contigo. ${ }^{15}$

14/ 07 / 2003, Línea de conversación:

"Asunción" / Paraguay

La mayoría de las veces, sin embargo, esas preocupaciones constituyen apenas un aspecto del interés por la seguridad personal al que se suman la ansiedad por detectar lugares en los que dólares y euros rinden más y la excitación suscitada por la abertura de nuevos mercados, para seleccionar las ciudades para visitar. Esos usuarios establecen una íntima asociación entre pobreza, sobre todo pobreza reciente, y el hecho de que nuevas mujeres se integren en el mercado del sexo. Los países que acaban de ser afectados por problemas económicos se tornan objeto de especial interés.

Leí que Montevideo y Uruguay están sufriendo por causa de la quiebra en Argentina. Hay muchas oportunidades allí. ${ }^{16}$

7/05/2002, Línea de conversación:

"Uruguay" / Montevideo 
En el comienzo de 2002, cuando la crisis económica en Argentina se agudizó, el país se convirtió abruptamente en "receptor" de un número creciente de turistas sexuales. A fines de la década de 1990, los periódicos argentinos trataban del turismo sexual como si fuera un problema absolutamente externo. Ya, en 2003, circulaban noticias que mostraban la alarma provocada por la presencia de turistas sexuales en el país. ${ }^{17}$ Prueba de la integración de Argentina en los circuitos mundiales de turismo sexual es su incorporación, a partir de ese período, en las páginas web destinadas a difundir informaciones para los turistas sexuales (en las que no aparecía hasta el año 2000). En esos espacios virtuales, la disponibilidad de las argentinas para ese tipo de turismo está abiertamente asociada a la crisis económica.

De acuerdo con la última encuesta, aproximadamente 50\% de la población de Argentina vive ahora oficialmente en la pobreza. Por otra parte, los argentinos están acostumbrados a una buena vida, sofisticada y no quieren abandonarla. Así que la solución obvia si eres una mujer joven y no muy fea: la prostitución. ${ }^{18}$

16/03/, Línea de conversación:

"Incredibly cheap sex in BA" / Argentina

La pobreza vinculada a las desigualdades regionales internas a cada país es registrada por los viajeros con mayor experiencia. En el caso de Brasil, los usuarios con mayor familiaridad con el país detectan el rechazo a los extranjeros, percibidos como inferiores, por parte de mujeres bien ubicadas en la escala de clases de las ciudades más ricas.

Te recomiendo que visites Sao Paulo... Hay que considerar algo importante: en Sanpa las mejores discotecas non pro típicamente están llenas de chicas de clase media y clase alta. Estas chicas le dan importancia a la CLASE. En otras palabras, aunque seas atractivo te ignorarán si no pareces pertenecer a la "clase" de ellas. Los turistas también hacen parte de esa categoría indeseable. ${ }^{19}$

02/03/2003, Línea de conversación: "Paulsobe en el Carnaval de 2003 en São Paulo" / Brasil

Los mongers con más experiencia hacen circular mapas de las diversas ciudades del mundo. Buenos Aires, en su nuevo boom ha 
sido objeto de descripciones rigurosas. Hay listas detalladas de saunas, casas de masaje, locales de strip tease, agencias de acompañantes. El intercambio de mensajes, en los que son evaluados países y regiones, permite trazar el camino que los usuarios afirman realizar por diversas ciudades del circuito mundial de turismo sexual, dando también una idea de las regiones preferidas. Los informes, que a veces adquieren formatos análogos a los de guías de viaje, sugieren una impresionante circulación entre los más diversos países. Usuarios que están en América del Sur parten para África, Asia, Oriente Medio. En lo que se refiere a América del Sur muestran el tránsito reiterado por diversos lugares. Los mismos viajeros circulan por Chile, Argentina, Brasil. Y vale observar que la integración de Argentina en el circuito mundial de turismo sexual muestra una curiosa circulación de viajeros en busca de sexo, análoga a la realizada por prostitutas extranjeras en el comienzo del siglo XX, en las narrativas sobre trata de ese período (Pereira, 2002). En los relatos de viaje de esos turistas, Río de Janeiro y Buenos Aires aparecen nuevamente ligadas en el contexto de la prostitución.

La correspondencia sugiere, también, la existencia de una intensa circulación de mujeres que ofrecen servicios sexuales. Chile, operando en el Cono Sur como Suiza lo hizo en la década del 90 en Europa, a la manera de polo de atracción de trabajadoras del sexo en función del valor de la moneda y las posibilidades de lucro, es presentado como el país privilegiado por trabajadoras del sexo de alto nivel de diversas naciones de América del Sur. Buenos Aires aparece como centro de prostitutas locales de alto nivel e inclusive, brasileñas y rusas, cobrando en torno de U\$40 por servicios de una hora, concentradas en agencias de escorts y en clubs y cafés de la Recoleta. Y mujeres vinculadas a niveles inferiores de prostitución son contactadas en los barrios de Constitución y Flores. Según los viajeros que transitan por Buenos Aires, son argentinas de las provincias, paraguayas y dominicanas. Otras ciudades son mencionadas ocasionalmente, Santiago del Estero, Córdoba y lugares tales como las Estancias para pescar. Pero la mayoría absoluta de los mensajes se refiere a Buenos Aires.

La correspondencia sugiere también que las mejores chicas de América del Sur pasan breves temporadas en Europa, una circulación considerada peligrosa, en la medida en que el contacto con mujeres y, particularmente, trabajadoras sexuales del Primer 
Mundo "des-latinoamericaniza" a las chicas, tornándolas más exigentes. Los mensajes permiten percibir cómo diversos factores se integran en las delimitaciones relevantes en términos de países, regiones y locales a ser visitados.

\section{Transgresiones}

En el web site algunos usuarios declaran, ocasionalmente, su predilección por fetiches específicos. Otros se enorgullecen de enseñar la utilización de juguetes a las nativas. Varios afirman disfrutar de prácticas levemente sado. El conjunto de los mensajes muestra el predominio de prácticas sexuales "universalmente" apreciadas (el BBBJ, sexo oral sin preservativo es considerado everyone's favorite). La habilidad en la realización de sexo oral y la disposición para el sexo anal se cuentan entre las prácticas favoritas, valorizadas por que consideran que las mujeres "Occidentales" se resisten a ellas. Para algunos viajeros, el sexo sin preservativo, posibilidad abierta generalmente en los lugares más pobres, aparece como factor de atracción. En ese sentido, y realizando reflexiones sobre los efectos de las campañas de salud destinadas, sobre todo, a las trabajadoras del sexo, Brasil y Argentina son considerados países difíciles. El SIDA es un tema que suscita preocupación en el site. Pero inclusive los viajantes que se preocupan por él aconsejan no exagerar, por lo menos en América del Sur. El uso del preservativo es considerado fundamental pero la tendencia general es pensar que el sexo oral no es tan peligroso y, además, observar que ciertos riesgos hacen parte del "deporte".

En el ámbito de América del Sur, otras particularidades tales como la existencia de fiestas, orgías con varias chicas, suelen estar vinculadas a las posibilidades abiertas por el Brasil y, de manera más específica, por Río, considerado la Meca del sexo profesional en esa región del mundo. Sin embargo, los aspectos que guían la circulación de esos turistas por el mundo no están asociados a prácticas sexuales específicas. La pobreza en sí no aparece como factor determinante en las elecciones, ni siquiera cuando es considerada extrema, como en Paraguay, país que, según esos viajantes ofrece el sexo más barato (real 3rd world) y las más amplias posibilidades de prácticas sexuales sin preservativo en el Cono Sur. 
Los intercambios de mensajes sobre los lugares percibidos como más exóticos, como Kenia, sugieren que, para algunos de esos viajeros, el sabor de lo que todavía es desconocido, virgen, no tocado por otros turistas, supera el valor concedido a prácticas sexuales. Algunos usuarios toleran comportamientos altamente restrictivos, inclusive tratándose de prostitutas, en aras de la obtención de placeres asociados a ese tipo de aventuras. Un experto en África describe sin trazos de irritación los hábitos y "limitaciones" de las chicas de los bares de Etiopía en lo que se refiere a posiciones y grados de desnudez.

Cambiar las posiciones también parece ser un problema, porque ellas prefieren el estilo misionero. A muchas no les gusta besar (recuerda, ellas piensan que vienes solo para eyacular) $\mathrm{y}$ algunas ni siquiera quieren que les beses los pezones o los cuerpos. Te daré un ejemplo bien vívido: si vas para pasar un tiempo corto con una chica, ella se quitará la ropa de la cintura para abajo, pero se quedará con la remera. Ella se negará a desvestirse completamente y esperará que estimules tu propio pene hasta que esté erecto, que te pongas el preservativo, la penetres y eyacules. ${ }^{20}$

26/05/2005, Línea de conversación:

"Ethiopia - The Ultimate Sex Guide"

Me detuve en las prácticas sexuales porque son sugestivas para pensar en el estilo de transgresión que permea los mensajes, que no está asociada a la búsqueda de sexo con niños ni a prácticas sexuales específicas o bizarras (kinky sex). En esos mensajes la idea de transgresión se delinea, sobre todo, en la posibilidad de escapar a las normas de relaciones consideradas "Occidentales" por esos viajeros. Se trata, básicamente, de evitar las relaciones estables, en situaciones igualitarias, contaminadas por compromisos emocionales.

En el web site, el mercado sexual global es presentado como marcado por diferencias substantivas, entre las que se destaca la manera en que las mujeres participan en él. La literatura sobre turismo sexual llama la atención por el hecho de que esa problemática se imbrica con la prostitución, pero no se reduce a ella (Oppermann, 1998; Davidson, 1998). En el site, hay distinciones frecuentes e importantes entre prostitutas (pros), no-prostitutas (non-pros) y semi-profesionales (semi-pros). Ciertas regiones del 
mundo son consideradas como eminentemente pobladas por prostitutas, como Pattaya, conocido resort de Tailandia. Al contrario, el acceso a no-prostitutas es percibido como prácticamente imposible en lugares como Buenos Aires y, en el Brasil, ciudades del Sur como Florianópolis y Curitiba, consideradas marcadas por la ascendencia europea, en las que las chicas no se deslumbran con los extranjeros.

Hay usuarios que valorizan la práctica del hobby con non pro's, porque posibilita sexo más "económico" y también porque proporcionan un plus de placer: con una pro se negocia, una non-pro presenta el desafío de tener que ser seducida. Para otros, la distinción entre las chicas no tiene importancia. Sin embargo muchos valorizan el acceso a una experiencia GFE (Girl friend experience) relacionándose con mujeres a las que se trata como novias, sin importar si son o no prostitutas. En esa situación, que no implica contrato de pago establecido por hora o por día, las chicas pasan días o semanas con los turistas. Estas situaciones son valorizadas porque además de ofrecer compañía, estimulan un cierto grado de afectividad (por parte de las chicas), percibido como importante para la entrega sexual plena. Sea cual sea la preferencia, en líneas de conversación de uno u otro país, se destaca permanentemente la importancia de mantener la distancia afectiva con las mujeres.

Elizabeth Bernstein $(2007,2001)$ ofrece algunos elementos para comprender el valor concedido a las experiencias GFE. De acuerdo con la autora, las transformaciones económicas de las décadas recientes reestructuraron la geografía social del trabajo sexual, en un proceso en el que participan el incremento del turismo y los viajes de negocios y también la intensificación del trabajo temporario y de las migraciones laborales a partir de los países "en desarrollo" para los "desarrollados". La industria del sexo se diversificó en este marco que, en los países del Norte, incluye una tensión. Se trata de, por un lado, la creciente aceptación del sexo como recreación, en el que el erotismo está libre de vínculos emocionales. Por otro, una presión normativa para retornar al sexo como relacional, inmerso en lazos amorosos. En esta última visión, presente en abordajes feministas, el sexo recreacional tiende a ser percibido como patológico. Ambas concepciones serían productos del mismo tiempo y lugar. En este proceso, los significados subjetivos presentes en las 
experiencias en el interior del mercado del sexo también se modificaron.

En el marco de un paradigma de comercio sexual postindustrial, la autora identifica un nuevo estilo de prestaciones sexuales, difundiéndose entre consumidores de sexo de clase media, predominantemente blancos, que frecuentan espacios cerrados en algunas ciudades del Norte. Se trataría de intercambios que involucran mucho más trabajo emocional y físico dentro del contexto comercial. En ellos se diluye la delimitación de espacios corporales y prácticas involucradas en otros tipos de canje de sexo por dinero. El nuevo estilo estaría caracterizado por una serie de elementos que remiten a las relaciones emocionales: el sexo como novios, los besos en la boca, las conversaciones y los masajes, y la utilización de períodos de tiempo más prolongados.

Parte significativa de la nueva variedad de trabajo sexual residiría en proveer "autenticidad delimitada", en vender y comprar conexión emocional y física temporaria, pero auténtica. Para algunas trabajadoras del sexo, esto requeriría satisfacer las fantasías de reciprocidad sensual de los clientes a través de la simulación de deseo, placer e interés erótico. Para otras podría involucrar el trabajo emocional y físico de producir lazos libidinales y emocionales genuinos, pero temporarios, dejando en los clientes la sensación de ser deseados, estimados y también amados. Este tipo de transacción introduciría elementos de la vida erótica "privada" y emocional en el ámbito del sexo comercial.

Bernstein argumenta que la interpenetración entre intimidad y comercio está transformando el carácter de esos dominios. Y si en el pasado la sexualidad vinculada al romance, presente en relaciones que tenían lugar en el espacio doméstico, adquiría sentido en el marco de una oposición ideológica con el mercado, las nuevas modalidades de sexo comercial no presentarían antagonismos con el comercio. Están disponibles para compra y venta como cualquier otra forma de actividad de ocio comercial.

Estas observaciones contribuyen para entender la búsqueda de relaciones GFE por parte de los turistas sexuales. Sin embargo, el trabajo de Bernstein fue realizado entre consumidores de sexo y trabajadoras sexuales de sectores sociales en los que el género aparece como la diferencia principal. Cabe preguntar sobre las nociones de transgresión que permean los encuentros sexuales entre hombres que, en el marco de la tensión entre sexo 
recreativo y relacional, optan por el consumo de sexo en viajes sexuales al "Tercer Mundo", en relaciones altamente desiguales con mujeres cuya posición social inferior se expresa en la imbricación entre género, nacionalidad, raza y clase y también cabe indagar sobre cómo esas nociones inciden en la creación de "nuevos" espacios de consumo.

\section{Fronteras}

¿Cuáles son las fronteras que delinean los lugares apreciados para este tipo de consumo de sexo? El conjunto de mensajes permite percibir que la atracción erótica vinculada a estilos de corporalidad asociados a ciertos lugares es un criterio de la mayor importancia. Esa correspondencia sugiere convergencias en lo que se refiere a rechazar la gordura femenina y la búsqueda de mujeres jóvenes, que no superen la franja de los 20 años. En el marco de esas convergencias, se eligen lugares en la base de distinciones que se expresan en clasificaciones, en una jerarquía construida en torno a la "calidad" de las mujeres, ítem en el que se integra la apariencia, juventud, el hecho de tener vaginas apretadas, motivo por el que privilegian aquellas que no fueron madres, y los estilos de sexualidad. El conjunto de esas cualidades está vinculado a características asociadas a regiones y nacionalidades, singularizados en una perspectiva comparativa. En esa relación, los estilos de corporalidad no son evaluados de manera uniforme.

Algunos usuarios dejan clara su preferencia por la blancura, corporificada por mujeres de los países del Norte, de pechos grandes. Pero ellas son consideradas inaccesibles para el tipo de relaciones que pretenden establecer. Los intercambios de mensajes de esos usuarios muestran como, en esos casos, las mujeres del "Tercer Mundo" operan a la manera de satisfacción sexual de segunda clase.

A partir de mi experiencia, creo que encontrarás el mayor número de chicas con pechos grandes en Europa, especialmente en países del Norte como Inglaterra, Holanda, Dinamarca, Alemania, Polonia... pero ellas no son prostitutas, así, lo único que puedo hacer es soñar con tocar esas cosas grandes y blandas. ${ }^{21}$

27/07/2002, Línea de conversación:

"Trip to Lima"/Peru 
Esos viajeros tienden a desvalorizar los tipos corporales asociados a África y a ciertos países de América del Sur, asociados a un grado extremo de pobreza que, según ellos, se expresa en la corporalidad, en la piel con acné, y en diferentes tipos de marcas o en la forma de pechos y colas.

He vivido en Nairobi (Kenya) durante los últimos 10 años y creo que estoy calificado para hablar sobre el asunto... Un paraíso? Bueno, sí, si no te importan los detalles. Si pretendes finura, discusiones filosóficas después del coito, rostros delicados y piel tersa, olvídalo! Lamentablemente [la mayoría de] estas chicas vive en pésimas condiciones de higiene -espera para verlas desnudas y te darás cuenta de eso: acné, cicatrices, heridas, marcas de todo tipo (" ¿Qué son esas cicatrices en tu pecho?" "La ex esposa de mi marido me mordió"-. Me las arreglé para acariciarla igual [estilo perrito], por causa del lado humorístico de la situación). Como siempre, lo que obtienes está en relación con lo que pagas... Todavía busco chicas blancas, el único lugar en que las encontré (damas asiáticas y no de los países occidentales) fue el "Cherry", en las tierras occidentales... Y si tienes alguna información sobre chicas occidentales aquí en Kenya, avísame por favor!!! 22

10/01 / 2001, Línea de conversación:

"Kenya" / Nairobi.

La valorización positiva de los cuerpos más "claros" es evidente también en los mensajes que tratan del Brasil, en los que se compara a las ciudades de Río y Sao Paulo. Esta última es descripta como ofreciendo mujeres más bellas y con gran diversidad, rubias y castañas, en cambio Río presentaría muchas más mulatas y negras que, a pesar de menos bellas, aparecen como más disponibles para el sexo. Los colores más obscuros, asociados a una hiper-sexualización "natural", pueden llegar a determinar la preferencia por ciertas regiones del mundo.

África Sub-Sahariana. Para ellos, el sexo no es algo sagrado, es parte de la vida cotidiana, como comer, beber y coger. La cultura también tolera e inclusive fomenta parejas múltiples. Un amigo mío describió así la actitud que tienen en relación al sexo: "un negro invita a una negra a hacer sexo con la misma facilidad con que un blanco invita a una blanca a tomar el té". ${ }^{23}$

12 / 10/2001, Línea de conversación: "Kenya" / Nairobi 
La atribución de estilos de sexualidad siempre es racializada. Sin embargo, en ciertas circunstancias, los aspectos que adquieren centralidad en estos procesos son los trazos culturales, naturalizados, asociados a ciertos países y/o regiones. Citando un handbook sobre Cuba, difundido entre los usuarios del web site, un monger afirma:

Cuba es una sociedad sexualmente permisiva... Hombres y mujeres cubanos están impregnados por un erotismo festivo que trasciende las salidas puritanas en Europa o América del Norte. La seducción es un pasatiempo nacional al que se dedican los dos sexos. La promiscuidad es desenfrenada... El amor no está asociado al sexo. Y los géneros son inusualmente atrevidos... Las cubanas no caminan, ellas se bambolean... La literatura cubana rebosa de referencias a "las nalgas cubanas", generalmente rollizas y perteneciendo a un mulato con cuerpo bien torneado. ${ }^{24}$

06/10/1999, Línea de conversación: Cuba.

En lo que se refiere a América Latina, la sexualización tiende a ser vinculada a una cierta "tropicalidad". Este aspecto queda claro en los mensajes relativos a los países andinos, en los que las regiones de la selva son preferidas a las del altiplano, percibidas como excesivamente indígenas y, por lo tanto, poco "calientes". En ese cuadro, los cuerpos femeninos, asociados a la flora y fauna regionales, adquieren los atributos de los animales con los que son comparadas.

Acabo de llegar de Lima, donde la pasé muy bien. Agarré una chica de la selva, una serpiente en la cama, con el cuerpo bien sólido y compacto. ${ }^{25}$

05/05/2003, Línea de conversación: “Trip to Lima” / Peru

Los procedimientos de sexualización también afectan a las latinoamericanas de piel considerada más clara, permitiendo percibir la diversidad de modalidades de racialización presentes en el turismo sexual. Es importante destacar que no se trata aquí del conocido procedimiento de "obscurecer" fenotipos "claros" mediante la asociación a alguna nacionalidad o región, como en los casos en que Brasil y sus habitantes, más allá de las tonalidades de piel, son considerados intrínsecamente morenos 
porque su nacionalidad ya les concede una filiación mestiza (Pontes, 2004; Beleli, 2005). Se trata de tornar inferiores mujeres cuyas pieles son percibidas como claras/caucásicas (un aspecto frecuentemente destacado), en la base de la atribución de trazos culturales o étnicos. Esos trazos, asociados a ciertas regiones, adquieren predominancia en la constitución de estilos de sexualidad.

Estos procedimientos adquieren sentido considerando los nuevos procesos de racialización mediante los cuales los migrantes de piel considerada clara son situados en posiciones sociales inferiores en Europa. Antes de explorar esa relación es necesario hacer una observación sobre la noción de racialización. En este momento, las perspectivas que utilizaron esa noción refiriéndose exclusivamente a los grupos desvalorizados por corporificar tonos de piel considerados más obscuros son problematizadas. Avtar Brah (1998), señalando que los procesos de racialización, históricamente específicos se basan en distintos marcadores de diferencia, afirma que negros y blancos tienen experiencias de género, clase y sexualidad mediante la "raza". Esto es obscurecido, a veces, porque la subjetividad blanca no siempre es aparente, porque "blanco" tiende a ser un significante de la dominación. Y, si en cierto momento la noción de racialización contempló los grupos desvalorizados por sus trazos fenotípicos (Anthias y Yuval Davis, 1993), los procesos recientes de racialización vinculados a las nuevas migraciones en Europa muestran procedimientos que sitúan en posición de desigualdad grupos cuyas pieles son consideradas claras (por ejemplo, con origen en el Este de Europa) tornándolos inferiores básicamente en términos étnicos o culturales. En esos casos la racialización no opera basándose en trazos fenotípicos sino a través de la naturalización de la etnicidad/nacionalidad que, atribuyendo trazos fijos a la cultura, retira a personas de pieles consideradas claras del lugar geopolítico asociado a la "blancura" (Silvernstein, 2005).

Al discutir las ventajas ofrecidas por Buenos Aires, los usuarios del web site muestran un procedimiento análogo en lo que se refiere a las mujeres de América del Sur. Esto es evidente en los mensajes de viajeros que aprecian los trazos europeos atribuidos a uruguayas y argentinas, percibidos como aspectos fenotípicos que se articulan a estilos de sexualidad latinos. 
Estas chicas son regias. Argentina y Uruguay son conocidos por las latinas que parecen Europeas -que combinación!! (No hay nada racista en esto, simplemente, en general, prefiero mujeres caucásicas, especialmente rubias). ${ }^{26}$

28/03/2001, Línea de conversación: "BA with pictures"

Dices que estas chicas parecen europeas pero cogen como Latinas? Que combinación! Ahora realmente quiero ir! ${ }^{27}$

17/09/2001, Línea de conversación: "BA with pictures"

La noche perfecta en Bs. As. Un paseo sin apuro por las calles del centro, una botella de buen vino con un bife fantástico, seguido por una ronda (o dos o tres) de conchas Euro-Latinas. ${ }^{28}$

20/09/2001, Línea de conversación: "BA with pictures"

Los mensajes sobre Argentina llaman la atención para los trazos "caucásicos" y para el grado de instrucción elevado de esas mujeres, una combinación que aparece incidiendo en el precio, tornándolas "caras" y, además, a diferencia de otras nativas de América del Sur, arrogantes "las más despiertas y manipuladoras en el mundo, todavía más que las rusas o ucranianas" (Línea de conversación: Attitudes, Changes and the Latin AAA Farm System). Usuarios con experiencia en el consumo de sexo en diversos países se quejan de la dificultad de establecer relaciones GFE con las argentinas, pues solo tienen acceso a profesionales "duras". Varios reclaman porque se trata de mujeres en la franja de los 30 años, prácticamente el doble de la edad de las que se consiguen en Colombia o Cuba. Otros registran como es incómodo estar con mujeres que fuman y mucho. Sin embargo, en el site crece el número de mensajes en los que las argentinas tienden a ser privilegiadas en la base de la estetización.

Acabo de estar en Río y debo admitir que creo que, en conjunto, las mujeres en BA son superiores en lo que se refiere al aspecto. ${ }^{29}$

18/03/2003, Línea de conversación: "Incredibly Cheap Sex in BA"

La belleza de esas mujeres, con aires europeos y, al mismo tiempo, portadoras de una latin sexuality (podría leerse como casi blancas), hace que Buenos Aires esté situada entre las 5 o 6 ciudades del mundo en las que la posibilidad de un encuentro con una beldad sea elevado, así como Chang Mai, Bangkok, 
Djakarta, Varsovia. Algunos viajeros prefieren a las brasileñas pues las consideran más "pasionales". Sin embargo, la mayoría de los usuarios que expresan sus opiniones sobre Argentina coincide en valorizar los estilos de corporalidad de piel clara asociados a las nativas del país.

Realmente creo que BA es más el lugar para mí. La piel oscura y los rostros de Río no me atraen tanto y estas chicas de BA, vi las fotos, parecen mucho más claras y más europeas. ${ }^{30}$

3/03/ 2005, Línea de conversación: "BA vs. Río?" / Argentina.

La apariencia europea me atrae más, por eso BA me parece mejor. Las chicas de BA parecen tener pechos bien dotados, más que la mayoría de las europeas. Lo mejor de las dos razas, las tetas grandes de las latinas y el aspecto claro de la europeas. $^{31}$

07/04 / 2005, Línea de conversación: “BA vs Río?” / Argentina.

A pesar de los aspectos "negativos", Buenos Aires continúa tornándose un destino favorito, en América del Sur, para muchos usuarios involucrados en el juego de una sexualización/ racialización que torna inferiores mujeres "parecidas" a las del Norte. Este estilo de relación con la diferencia ofrece elementos para comprender la veloz integración de Argentina en los circuitos mundiales de turismo sexual. Ella está, sin duda, vinculada a la crisis económica, a la desvalorización del dólar en el país, que facilita el acceso a servicios sexuales en una ciudad considerada más "segura" que otras en el Tercer Mundo, con excelente comida y bebida, y esos son aspectos comentados con frecuencia por los turistas sexuales. Pero esa integración está asociada, sobre todo, a la valorización de un estilo de sexualidad considerado como "caliente", corporificado por mujeres cuyo aspecto las aproxima a aquellas que tienden a ser las más valorizadas: las blancas e inaccesibles mujeres de los países del Norte.

\section{Conclusión: Geografías mutantes}

Este site, estimulando la búsqueda de una "autenticidad" turística corporeizada en mujeres de diversas regiones del mundo, 
posibilita la materialización del contacto sexual entre viajeros en busca de sexo y mujeres nativas. Funcionando como espacio de socialización colectiva y de producción de nociones racializadas y sexualizadas de mujeres del "Tercer Mundo" orienta, en escala global, la recreación de códigos de sexualidad y masculinidad asociados a la supremacía blanca y a una cierta idea de lo "Occidental". En este sentido, el análisis de los mensajes intercambiados por los usuarios refuerza las conclusiones de innumerables investigaciones sobre turismo sexual en diversas regiones del mundo. Sin embargo, el material, y esta es una de sus singularidades, muestra de manera particularmente nítida la relevancia que la práctica del sexo en relaciones cálidas y afectuosas, pero delimitadas por el intercambio del mercado adquiere en la manutención de las desigualdades presentes en este tipo de consumo de sexo.

La vinculación entre sexo y sentimientos aparece como una amenaza para ese estilo de masculinidad. Los viajeros en busca de sexo tratan de huir de esa amenaza, que se inicia en sus países de origen, mediante el consumo de sexo en "otras" partes del mundo. Considerando la insistencia en el tema, los llamados de atención sobre el peligro potencialmente ofrecido por los encuentros sexuales, inclusive por los abiertamente comerciales, sea en Tailandia, Cuba, Brasil o Argentina, cuando se confunde la naturaleza de las interacciones, tal vez sea una amenaza de la cual ni siquiera este tipo extremo de viajeros logra escapar.

El material analizado permite percibir que las alteraciones en la geografía de los circuitos mundiales de turismo sexual están, en el marco de ciertas condiciones económicas, asociadas a la imbricación entre trazos étnico/regionales y estilos de sexualidad que operan a la manera de atracción para el surgimiento de nuevos lugares de consumo de sexo. A pesar de ser siempre racializados, esos estilos no son homogéneos. En este marco coexisten distintos procesos de racialización, que participan en la sexualización de diferentes estilos de corporalidad. En lo que se refiere a Brasil y a Cuba, los mensajes remiten a estilos de feminidad asociados a una intensa sensualidad vinculada al color moreno. Pero en ese mismo marco surgen los mensajes que construyen Buenos Aires como nuevo destino para el consumo de sexo, habitado por mujeres caras, duras y exigentes, pero portadoras de una casi-blancura que envuelve, sin llegar a ocultar, un temperamento "caliente". 
Los turistas en busca de sexo que privilegian a las argentinas sexualizan/racializan mujeres cuyo fenotipo ofrece la atracción de una cierta proximidad, proporcionando la ilusión de situar en posición inferior mujeres próximas a aquellas (deseadas e inaccesibles en las condiciones de distancia afectiva y desigualdad por ellos exigidas) de sus países de origen.

\section{Notas}

1 Antropóloga, investigadora y académica de Núcleo de Estudos de Gênero-Pagu/Unicamp, Sao Paulo, Brasil.

2 Presento los términos Tercer Mundo, Norte, Sur y países ricos entre comillas, considerando que se trata de categorías problemáticas $\mathrm{y}$, sin embargo, necesarias para aludir a las desigualdades estructurales entre regiones y países.

3 Agradezco a Lea Fletcher la traducción de partes de este texto.

4 En los últimos años, los estudios sobre turismo sexual heterosexual han contemplado también los viajes de mujeres de los países ricos a regiones pobres del mundo con el objeto de consumir sexo ofrecido por los hombres nativos (Pruitt e Lafont, 1995; Meisch, 1995; Sánchez Taylor, 2001; Kempadoo, 2004; Frohlick, 2007; Cantalice, 2009). Sin embargo, hasta el momento no tengo conocimiento de sites de la web destinados a esas turistas.

5 Esos trabajos son escasos y están basados, sobre todo, en dos sites: Travel and the Single Male y The World Sex Guide.

6 Esa página web fue escogida por la enorme riqueza del material que difunde y porque fue recurrentemente mencionada por viajantes en busca de sexo entrevistados en una investigación anterior, sobre turismo sexual internacional, realizada en Fortaleza, capital del estado de Ceará, en el Nordeste del Brasil (Piscitelli, 2004; 2007; 2006).

7 Este texto está basado en una investigación apoyada por la FAPESP y el CNPq, agencias financiadoras del Brasil, entre 2003 y 2006.

8 Se discute la dificultad de obtener datos sobre los parámetros de la población involucrada, las limitaciones en relación a las entrevistas on-line, las interpretaciones equivocadas a las que puede conducir el hecho de trabajar exclusivamente en un medio textual (Hamann, 1997).

9 De acá en adelante las traducciones son de la autora. Women in western countries are spoiled bitches. They get far too much and give too little. They know they can treat their men like 
shit. Do you know why a woman smiles on her Wedding day? She knows she will not have to give blowjobs.

10 I am also so glad that there is a border between North and South America, as I would not want the American females to corrupt the Latin woman. They are a true work of art, and deserve to be loved and treated well.

11 La página web incluye mensajes, en número infinitamente menor, sobre consumo de sexo en países de Europa y América del Norte. Esas regiones no fueron incluidas en este cálculo, realizado sobre la base del total de mensajes intercambiados sobre cada uno de los países de América Central, del Sur y Caribe, África y Asia.

12 Vale recordar los períodos relativos a esas guerras: Indochina, 1946-1954; Corea, 1950-1953 y Vietnam, 1958-1975.

13 Until two years ago I always viewed mongering as a necessary evil. It was always an activity I had to enjoy alone, that I could not talk about. Other guys would talk about football or cars, I'd be thinking of pussy. I joined this board at the same time I took my first Rio trip. I have changed dramatically, knowing there is place in the world with probably tens of thousands of beautiful working girls and thousands of fellow mongers like me.

14 I saw something interesting in Diani Beach south of Mombasa on the coast. I saw two European women (one was drop dead gorgeous) with male prostitutes - tall black Masai men dressed up in their traditional garments. If I had had my camera with me I would have taken a picture of them and posted it here. I had read about this before, but it was the first time I have actually seen it in real life.

15 Anyway guys, even if the legal age of consent would be 5, i think that it is immoral to have money-sex with a girls under $18 \ldots$ for the simple reason that a girl under a certain age is not able to decide in a free way if she wants to have sex with you...

16 I read Montevideo and Uruguay are hurting from the Argentina crash. Many opportunities there.

17 Caparrós, Martín. Viajando se conoce gente. El turismo sexual: una costumbre de fin de siglo. Revista del Clarín, junio de 2000; DelgaDO, Daniel. Los turistas buscan sexo en Buenos Aires. Qué ciudad acogedora. TXT, año 1, n 47, Buenos Aires, 06/02/2004; El turismo sexual y sus falsas promesas para marear a chicas pobres. Clarin, 16/02/2004.

18 According to the latest survey, close to $50 \%$ of the population in Argentina live now officially in poverty. On the other hand, Argentinians are used to a good and sophisticated life they are not easily willing to give up. So the obvious solution if you are young and not too ugly woman: prostitution.

19 I recommend that you visit São Paulo... One important thing to 
consider: the top non-pro dance clubs in Sampa are typically filled with middle to upper class girls only. For these girls CLASS matters. In other words, even if you are a good looking guy but seem like you don't belong to their "class", they will ignore you, Tourists fall into this non-wanted category as well

20 Changing positions also seems to be a problem, as they prefer only missionary. Many wouldn't like kissing (remember, they think you came only in order to ejaculate) and some wouldn't even like to have their nipples/bodies kissed. I should give you a very vivid example: if you go with a girl for a short time, the lady will undress from the waist down, but will remain in $\mathrm{t}$-shirt. She will refuse to be fully undressed and she will be expecting that you stimulate your penis until it will become erected, put condom, fuck her and ejaculate.

21 From my experience, I believe that you will find the biggest number of big breasted girls in Europe, especially in Northern countries like England, Netherlands, Denmark, Germany, Poland... but these are not prostitutes though, so the only thing I can do is dream about touching those soft biggies!...

22 I have been living in Nairobi (Kenya) for the last 10 years now, and I guess I am pretty qualified to speak on the matter... A paradise? If you don't care about the details, yes. If you pretend finesse, post-coital philosophical discussions, delicate faces and smooth skin, forget about it! Unfortunately [most of] those girls live in dramatically low hygiene conditions - just wait to see them naked to realize this: acne, scars, wounds, marks of all kind ("What are all those scars on your chest?" "The former wife of my husband bitted me" - I managed to fondle her anyway [doggy style] just because of the humorous side of the situation). You get what you paid for, as usual... I am still looking for white girls, the only place where I found them (Asian ladies, not from western countries) was the "Cherry", in Westlands... And, if you have any information on Western girls here in Kenya, please let me know!!

23 Sub-Saharan África. Sex is not a sacred thing for them, it is a part of daily life - like eating, drinking and fucking. Their culture also tolerates multiple partners and even encourages it. A friend of mine described their attitude to sex as "a black man will ask a black woman for sex as easily as a white man would ask a white woman for a cup of tea".

24 Cuba is a sexually permissive society... Cuban men and women alike pervade a joyous eroticism that transcends the hang-ups of essentially puritanical Europe or North America. Seduction is a national pastime pursued by both sexes. Promiscuity is rampant... Love is not associated with sex. And both genders are unusually bold... Cuban women don't walk, they sway... Cuban literature 
overflows with references to "las nalgas cubanas" - the Cuban ass - usually plump and belonging to a well rounded mulatto.

25 I just came back from Lima, and I had a great time. I took one girl from la selva, a snake in bed, very solid body and compact. Trip to Lima/Peru.

26 These chicks are great. Argentina and Uruguay are known for hot Latinas with European looks - what a combo!! (Nothing racist intended here, I simply generally prefer Caucasian women, blondes especially).

27 So you are saying these girls look like Europeans but fuck like Latinas? What a combination! Now I really want to go!

28 The perfect night in Bs. As.. A leisurely stroll down the streets in the center, a bottle of fine wine with a fantastic steak, followed by a round (or two or three) of Euro-Latina pussy.

29 I have just been in Rio and must admit that I think overall the women in BA are superior in looks.

30 I really think BA is more the place for me. I don't so much dig the Rio faces and darker skin and these BA girls I have seen pictures of look a lot fairer and more European

31 I am attracted to the European look more myself, so BA looks better to me. The girls in BA look to have well endowed chest, where as most Europeans do not. The best of both races large tits of Latins and the fair look of Europeans

\section{Bibliografía}

Agustin, Laura: Sex at the margins. Migration, Labour Markets and the Rescue Industry. Londres: Zed Books, 2007.

Anthias, Floya y Nira Yuval-Davis. Racialized Boundaries. Race, nation, gender, colour and class and the anti-racist struggle. Londres: Routledge, 1993.

Aparicio, Frances y Susana Chaves-Silverman. Tropicalizations. Transcultural representations of Latinidad. New England: Dartmouth College, University Press of New England, 1997.

Appadurai, Arjun. Modernity at large. Cultural Dimensions of Globalization. Minnesota: University of Minnesota Press, 1996.

Beleli, Iara. "A marca Brasil". Anales del I Congreso Latinoamericano de Antropología, Universidad Nacional de Rosario. CD (2005).

Bernstein, Elizabeth. Temporarily Yours. Intimacy, Authenticity, and the Commerce of Sex. Chicago: The University of Chicago Press, 2007.

----- "The Meaning of the Purchase: Desire, Demand and the Commerce of Sex". Ethnography. 2 (2001): 389-420.

Bishop, Ryan y Lillian Robinson. “Travelers' Tails: Sex diaries of tourists 
returning from Thailand." En: Thorbek, Susanne y Bandana Pattanaik. Transnational prostitution. Changing global patterns. Londres: Zed Books, 2002.

Brah, Avtar. Cartographies of diaspora, contesting identities. Londres: Routledge, 1996.

Brennan, Denise. What's Love Got to do with it? Transnational Desires and Sex Tourism in the Dominican Republic. Durham: Duke University Press, 2004.

Cantalice, Tiago. "Dando um banho de carinho!. Os caça gringas e as interações afetivo-sexuais em contextos de viagem turística (PipaRN)". Dissertação de mestrado defendida no Programa de PósGraduação em Antropologia da Universidade Federal de Pernambuco, Recife, 2009.

Cavanagh, Allison. "Behaviour in Public?: Ethics in Online Ethnography." Cybersociology, 1999 http: / / www.cybersociology.com

Chow-White, Peter. "Race, gender and sex on the net: semantic networks of selling and storytelling sex tourism." Media, Culture and Society. 28 (2006): 883-905.

Davidson, Julia O'Connell. "Sex Tourism in Cuba". Race and Class. 38 (1996): 39-48.

----- "Prostitution, Power and Freedom". Michigan: Cornwall, The University of Michigan Press, 1998.

Dias Duarte, Luiz Fernando. "A sexualidade nas ciências sociais. Leitura crítica das convenções". En: Piscitelli, Adriana, et al. Sexualidades e saberes, convenções e fronteiras. Río de Janeiro: Garamond, 2004.

Enleo, Cynthia. Bananas, beaches and bases. Making feminist sense of International Politics. Berkeley: University of California Press, 1989.

Escobar, Arturo. "Welcome to Cyberia: Notes on the Anthropology of Cyberculture. Current". Anthropology. Vol. 35, 3 (1994): 211-233.

Frohlick, Susan. "Fluid Exchanges: The negotiation of Intimacy between Tourist Women and Local Men in a Transnational Town in Caribbean Costa Rica". City and Society. Vol. 19, 1 (2007): 139-168.

Gauntlett, David. "Digital Sexualities: a guide to internet resources". Sexualities. Vol 2, 3 (1999): 327-332.

Guimarães, Mário Jr. “O Cyberespaço como Cenário para as Ciências Sociais". Revista de Antropología. Vol. 2, 1 (2000): 139-155.

Hamman, Robin. "The application of Ethnographic Methodology in the Study of Cybersex". Cybersociology, 1997 http:/ / www.cybersociology.com

Hine, Christine. Virtual Ethnography, Londres: Sage, 2000.

Kempadoo, Kamala. Sexing the Caribbean, gender, race and sexual labor. New York: Routledge, 2004.

Maccannell, Dean. The tourist, a new theory of the leisure class. New York: Shocken, 1989. 
Meisch, Lynn. "Gringas and Otavaleños, Changing Tourist Relations." Annals of Tourism Research. Vol. 22, 2 (1995): 441-462.

Michel, Franck. "Le tourisme sexuel en Asie: du cauchemar à l'horreur." En: Michel, Franck (éd.) Tourismes, touristes, sociétés. Paris: Éditions L’Harmattan, 1998.

Mullings, Beverly. "Globalization, tourism, and the International Sex Trade." En: Kempadoo, Kamala. (ed.) Sun, Sex and Gold, Tourism and Sex Work in the Caribbean. Maryland: Rowman and Littlefield, 1999.

Nagel, Joane. Race, ethnicity and sexuality. Intimate Intersections, Forbidden Frontiers. Oxford: University Press, 2003.

Oppermann, Martin. Sex Tourism and prostitution, aspects of leisure, recreation and work. New York: Cognizant Communication Corporation, 1998.

Pereira, Cristiana Schettini. "A vida na janela: uma história social da prostituição no Rio do Janeiro contemporâneo". Tese de doutoramento. Unicamp, Campinas, 2002.

Piscitelli, Adriana. "Shifting Boundaries. Sex and Money in the Northeast of Brazil." Sexualities. Vol 10, 4 (2007): 489-500.

----- "Transnational sex travels: negotiation identities in a tropical paradise". Texto presentado en el Seminario Translocalities/Translocalidades: feminist politics of translation in Latin America, Amherst: Center for Latin American - Caribbean and Latin Studies, 2006.

----- "On Gringos and Natives, gender and sexuality in the context of international sex tourism in Fortaleza." Vibrant - Brazilian Virtual Anthropology. 1 (2004).

------ "Exotismo e autenticidade: relatos de viajantes à procura de sexo." Cadernos Pagu. 19 (2002): 195-233.

Pontes, Luciana. "Mulheres brasileiras na mídia portuguesa." Cadernos Pagu. 23 (2004): 229 - 257.

Pruitt, Deborah y Suzanne Lafont. "For love and money. Romance Tourism in Jamaica." Annals of Tourism Research. Vol. 22, 2 (1995): 422-440.

Rago, Margareth. “Globalização e imaginário sexual, ou 'Denise está chamando'." Enero, 2008 http://lite.fae.unicamp.br/grupos/ geish/geirevis2.html

Rivers-Moore, Megan. Getting ahead in Gringo Gulch: Transnational Sex Tourism in Costa Rica. Phd dissertation, University of Cambridge, 2009.

Sánchez Taylor, Jacqueline. "Dollars are a girl's best friend? Female tourists' sexual behaviour in the Caribbean." Sociology. Vol. 35, 3 (2001): 749-764.

Seabruck, Jeremy. Travels in the skin trade. Tourism and the Sex Industry. Londres: Pluto Press, 2001.

Silva, Ana Paula y Thaddeus Blanchette. "Prostituição e namoros inter- 
nacionais em Copacabana." Anales del I Congreso Latinoamericano de Antropología, Universidad Nacional de Rosario. CD, 2005.

Silverstein, Paul A. "Immigrant Racialization and the New Savage Slot: Race, Migration, and Immigration in the New Europe." Annual Review of Anthropology. 34 (2005): 363-84.

Strathern, Marilyn. "Comments on Welcome to Cyberia." Current Anthropology. Vol. 35, 3 (1994): 211-233.

Thorbek, Susanne y Bandana Pattanaik. Transnational prostitution. Changing global patterns. Londres: Zed Books, 2002.

Wittel, Andreas. "Ethnography on the Move: From Field Net to Internet." Qualitative Social Research. Vol. 1, 1 (2000). 